\title{
Penetration and Splitting of Optic Nerve by Tuberculum Sellae Meningioma
}

\author{
Seong-Cheol Park, M.D., M.S., ${ }^{1}$ Sang Hyung Lee, M.D., Ph.D. ${ }^{1,2}$ \\ Department of Neurosurgery, ${ }^{1}$ Seoul National University College of Medicine, Seoul, Korea \\ Department of Neurosurgery, ${ }^{2}$ Seoul Metropolitan Government-Seoul National University Boramae Hospital, Seoul, Korea
}

Preservation of the optic nerves is an important issue in the resection of tuberculum sellae meningiomas. We report the case of a patient whose optic nerve was penetrated by a tuberculum sellae meningioma. During surgery, a bulging tumor was found to penetrate the right optic nerve. The tumor was gross totally removed, including tumors bulging through the optic nerve. Two trunks of the split optic nerve were preserved. The penetrated shape of the optic nerve may lead to misjudgment and its damage. Divided trunks of optic nerves are difficult to recognize and may be confused for the tumor capsule, because they may be thinned and seem to contain tumors. In addition, a single trunk may be confused for the whole nerve; thus, the other trunk may be easily damaged if not dissected cautiously. Treatment strategy according to the remnant visual acuity was suggested.

Key Words : Meningioma · Optic nerve · Penetration · Visual acuity.

\section{INTRODUCTION}

In patients with tuberculum sellae meningioma, preservation of the optic nerve is one of the most significant issues ${ }^{12)}$. We report the rare case of a patient whose optic nerve was penetrated by a meningioma. Such cases require special attention in order to preserve the optic nerves.

\section{CASE REPORT}

A 68-year-old woman presented with decrement of visual acuity of the right eye 1 year ago. Magnetic resonance (MR) imaging revealed a tuberculum sellae meningioma (Fig. 1). The tumor was enhanced with homogeneous intensity after administration of a contrast material. After a year, she experienced complete loss of visual acuity of the right eye without light sense. Her visual acuity on the left side also began to decrease to 0.4 and the visual field reduced severely. At this point, we undertook surgery.

The tumor was removed through a right pterional approach. The subfrontal route was mainly used to resect the tumor. After retraction of the right frontal lobe, both the tumor and the superiorly displaced right optic nerve trunks penetrated by the tumor were exposed. However, we could not recognize the penetrated optic nerve for a while. It appeared as parts of the tumor because the tumor lay in the middle of the penetrated optic nerve and the two penetrated trunks were thin (Fig. 2A). The tumor was attached to the tuberculum sellae, which was considered as the site of origin confirming the diagnosis of a meningioma originated from the tuberculum sellae. The tumor was dissected from the adjacent cortices and nerves by maintaining arachnoid planes. The whole course of the nerve could be visualized with the removal of most of the tumor.

To confirm the penetration, the operator biopsied the bulged tumor (black arrow, Fig. 2A) penetrating the optic nerve. The tumor around the optic nerve was resected gross totally without tumor enhancements in postoperative MRIs (Fig. 3). The remaining preserved optic nerve had a penetration after tumor resection (black arrow, Fig. 2B). Pathological examination revealed the absence of neural tissue in the bulging tumor between the nerve trunks. No neural or glial tissue was found in the biopsy sample, and the pathologies of the main tumor mass and the bulging tumor were identical-grade I meningothelial meningioma with a Ki-67 index of $2 \%$. Postoperative course was uneventful and blindness in the right eye was unchanged. However, visual acuity of the left eye improved slightly to 0.6 and its visual field improved within 1 month post-operation (Fig. 4).

- Received : October 27, $2014 \cdot$ Revised : July 7, $2015 \cdot$ Accepted : August 6, 2015

- Address for reprints : Sang Hyung Lee, M.D., Ph.D.

Department of Neurosurgery, Seoul Metropolitan Government-Seoul National University Boramae Medical Center, 20 Boramae-ro 5-gil, Dongjak-gu, Seoul 07061, Korea

Tel : +82-2-2072-2352, Fax : +82-2-744-8459, E-mail : nslee@snu.ac.kr

- This is an Open Access article distributed under the terms of the Creative Commons Attribution Non-Commercial License (http://creativecommons.org/licenses/by-nc/3.0) which permits unrestricted non-commercial use, distribution, and reproduction in any medium, provided the original work is properly cited. 
No additional events or symptom aggravation were noted at the 1-year follow up.

\section{DISCUSSION}

To our knowledge, penetrated optic nerves have been reported in about 10 cases of aneurysms ${ }^{1-4,8-10,16,17)}$. Thus, their inci-
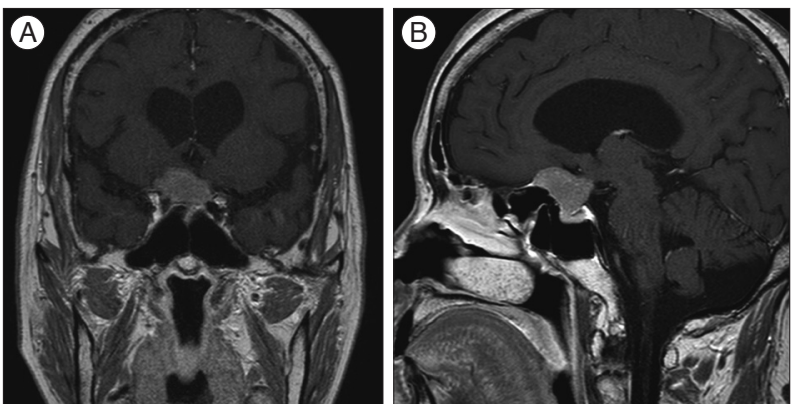

Fig. 1. Preoperative coronal (A) and sagittal (B) T1-weighted enhanced magnetic resonance images of the tuberculum sellae meningioma. Optic nerve penetration could not be identified in preoperative images.
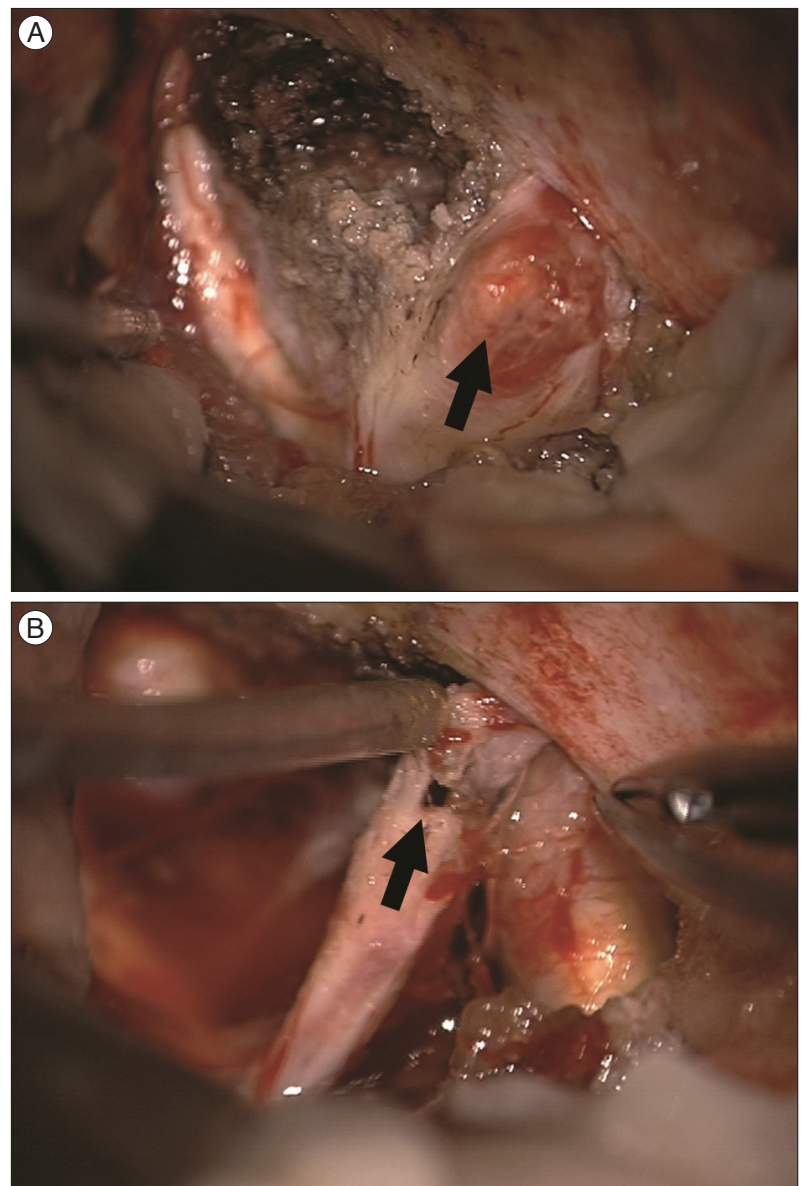

Fig. 2. Introperative images (A) bulged tumors (black arrow) through the two trunks of the right optic nerves were found. (B) Penetration into the optic nerve during tumor resection. There were remnant tumors between the suction tip and optic canal and between the optic nerve and internal carotid artery. These tumors were further removed gross totally. The tumor was whitish with low vascularity. dence seems rare. Optic nerve penetrations by meningiomas or other tumors are rarer, with only 3 cases reported in 2 studies ${ }^{5,14)}$. The cause of penetration of the optic nerve was probably tumor invasion in those cases. In the present case, our patient was op-
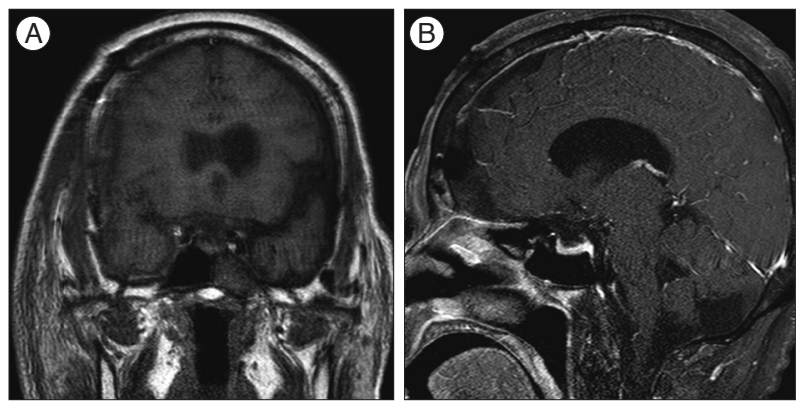

Fig. 3. Postoperative coronal (A) and sagittal (B) T1-weighted enhanced magnetic resonance images. No remnant tumor was identified.
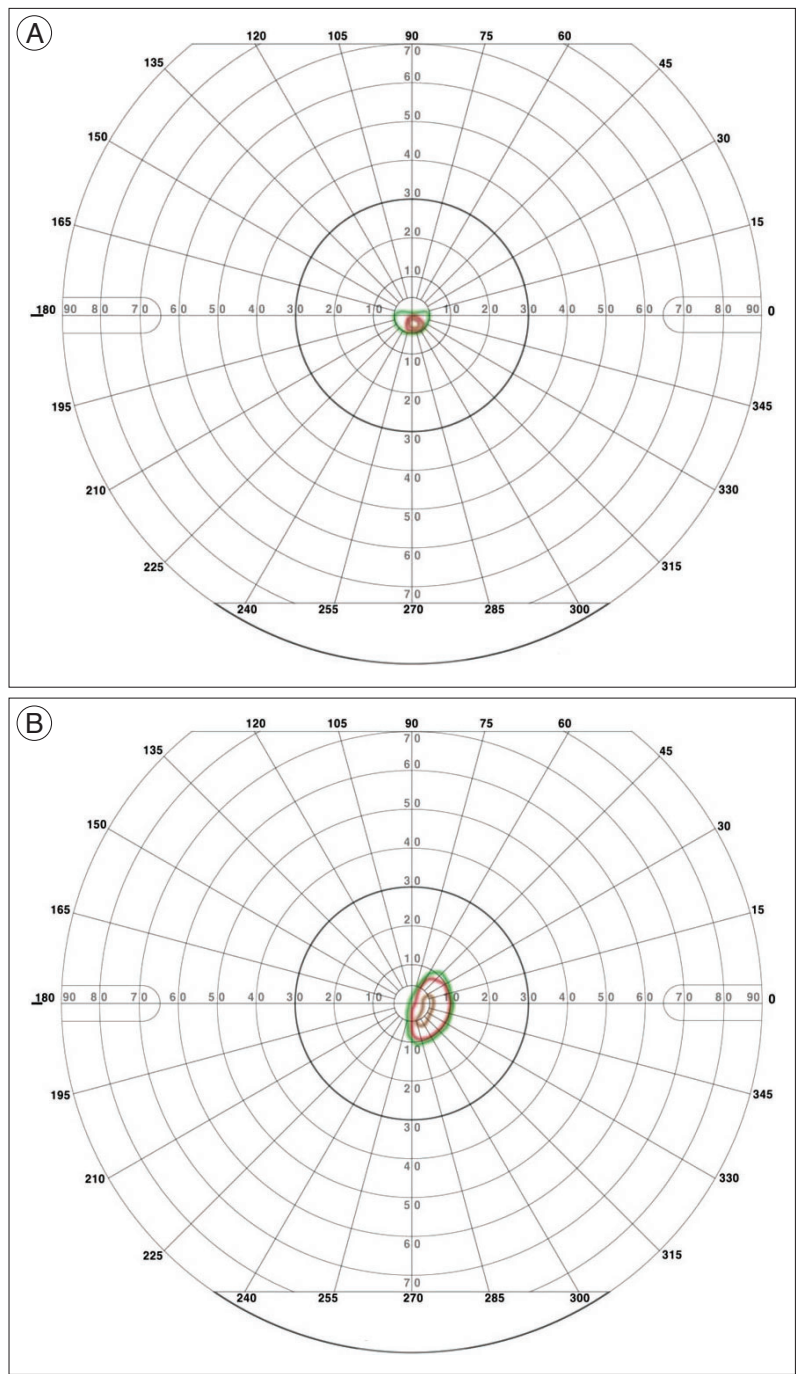

Fig. 4. Preoperative visual field of the left eye 1 month before the operation $(A)$ and postoperative visual field of the left eye 1 month after the operation (B). Outermost green line indicates the visual field that can identify a $64-\mathrm{mm}^{2}$ sized object. Inner two red lines indicate visual fields that can identify $4-\mathrm{mm}^{2}$ and $1 / 4-\mathrm{mm}^{2}$ sized objects. The right eye was blind and a visual field examination was impossible. 
erated later than that previously reported. Therefore, optic nerve penetrations in this patient could be a rare and late finding of a progressed tuberculum sellae meningioma where the growing mass might have intruded into the slit of the optic nerve ${ }^{5,14)}$. In general, optic nerve penetration by a tumor is thought to be very rare because optic nerve compression becomes symptomatic early in the course of the tumor, which is usually operated much before the actual penetration. However, there are cases reported wherein the tumor penetration resulted in remnant visual acuity; in such cases, a congenital or de novo splitting of the optic nerve has been suggested ${ }^{5,6)}$.

In the patient reported here, the right eye was already blind suggesting severe optic nerve damage; therefore, preservation of the right optic nerve was less important. However, patients in the previous two reports with penetration of the optic nerves by tumors had remnant visual function. Therefore, preservation of the penetrated optic nerve was justified in those cases ${ }^{5)}$. In cases of a flattened oculomotor nerve severely adhered to a meningioma similar to that seen in our patient, even a meticulous and gentle dissection can damage nerve function and have unsatisfactory results. Penetration of the optic nerve is especially confusing, and can be a surgical challenge. Bulging tumors and a small split nerve make it very difficult to locate the optic nerve; this condition forces the surgeon think that he/she is dissecting around the tumors, not the other trunk of the optic nerve, thereby increasing the risk of intraoperative optic nerve damage. In one study, encasement of the internal carotid artery affected the resection grade ${ }^{11)}$. Thus, penetration of the optic nerve could be a factor affecting the tumor-resection grade especially when vision is still functional. In addition, involvement of the meningioma was a factor related to post-surgical visual acuity decrement ${ }^{11)}$. Optic nerve penetration may also be a poor prognostic factor of visual acuity. We discussed treatment plans according to the remnant visual acuity and dissection plane between the tumor and optic nerve.

\section{Preserved dissection plane between the tumor and optic nerve with remnant visual function}

In cases with remnant visual function of the involved optic nerve, careful observation of the nerve contours is essential to prevent inadvertent damage. Recognizing the penetrated structure is important for proper dissection following the interface between pathologic and normal structures with an anatomic distortion. Therefore, we suggest that removal of tumors adjacent to and penetrating the optic nerve should be started after identifying the entire optic nerve trunk. This will help avoid damaging the nerve in both ordinary and split cases, especially when vision is functional. In a meningioma study with optic nerve involvement, careful dissection from the bone margin was recommended and suggested to be useful ${ }^{7)}$. In our surgery, both trunks of the thinned split optic nerve could be dissected from the inferiorly located tumor at first from the bone margin anteriorly. After identifying the entire optic nerve, the tumor bulging through the penetration was removed. Our experience and previous reports suggest that careful surgical resection without damaging optic nerve structures is possible especially when the surgeon identifies the complete nerve structure including both trunks around the tumor penetration (Fig. 2B) ${ }^{15)}$.

\section{Adherence between the tumor and optic nerve with remnant visual function}

In the case of optic nerve-penetrating tumors lying close to the nerve with remnant visual function, radical dissection and removal may not be beneficial for visual acuity. Therefore, partial removal and postoperative radiation therapy may be an option. For example, combined surgical resection and radiosurgery were used to treat an optic nerve sheath meningioma (a meningioma close to the optic nerve $)^{7,13)}$. Conformal radiosurgery or fractional stereotactic radiosurgery are possible options for maintenance of visual acuity ${ }^{7,13)}$. In cases of split optic nerves, a portion of the tumor penetrating the optic nerve may be very difficult to treat selectively by stereotactic radiosurgery without damaging the optic nerve. This is because even considering the resolution of stereotactic radiosurgery that depends on the smallest collimator $(4 \mathrm{~mm})^{7}$, almost the same dose would enter both the penetrating tumor and the penetrated optic nerve. To overcome this problem, fractionated stereotactic radiosurgery may be an option for meningiomas penetrating the optic nerve ${ }^{7)}$.

\section{No remnant visual function}

Our patient experienced loss of functional visual acuity in the penetrated optic nerve. In the case of a non-functional optic nerve, we speculate that a more aggressive surgical resection would be the best option. We preserved the optic nerve structure in our case and could remove the tumor completely (Fig. 3) without damaging the optic nerve (Fig. 2B). However, visual function did not return. Therefore, the optic nerve may be sacrificed when visual function is absent for a long time in split optic nerve cases, especially when the optic nerve hinders complete removal of malignant or clinically aggressive tumors.

\section{CONCLUSION}

Preserving an optic nerve may be more difficult in the case of penetration. Although optic nerve penetration by a tumor is rare, such cases help us reconsider the best surgical strategy for tumor removal in relation to the optic nerve and visual function.

\section{References}

1. Beatty RA : Splitting of the optic nerve by a carotid-ophthalmic artery aneurysm. Case report. J Neurosurg 65 : 560-562, 1986

2. Date I, Akioka T, Ohmoto T : Penetration of the optic chiasm by a ruptured anterior communicating artery aneurysm. Case report. J Neurosurg 87 : 324-326, 1997

3. Feliciano CE, Samson D : Split optic nerve penetrated by a carotid-ophthalmic aneurysm another instance of a rare presentation : a case report. IJCRI 3 : 39-44, 2012 
4. Fujita A, Tamaki N, Yasuo K, Nagashima T, Ehara K : Complete penetration of the optic chiasm by an unruptured aneurysm of the ophthalmic segment : case report. Surg Neurol 57 : 130-134, 2002

5. Gagliardi F, Boari N, Losa M, Mortini P : Longitudinal splitting of optic pathways by non-functioning pituitary macroadenoma : report of two cases. Acta Neurochir (Wien) 152 : 1065-1068; discussion 1068, 2010

6. House PA, Couldwell WT : De novo fenestration of the optic nerve. Case illustration. J Neurosurg $102: 390,2005$

7. Jallo GI, Benjamin V : Tuberculum sellae meningiomas : microsurgical anatomy and surgical technique. Neurosurgery 51 : 1432-1439; discussion 1439-1440, 2002

8. Jea A, Başkaya MK, Morcos JJ : Penetration of the optic nerve by an internal carotid artery-ophthalmic artery aneurysm : case report and literature review. Neurosurgery 53 : 996-999; discussion 999-1000, 2003

9. Joo SP, Kim TS : Splitting of the optic nerve associated with ruptured anterior communicating artery aneurysm. Neurol Med Chir (Tokyo) 47 : 501-502, 2007

10. Kanamaru K, Ishida F, Taki W : Splitting and penetration of the optic nerve by an aneurysm arising from the anterior wall of internal carotid artery : case report. J Neurol Neurosurg Psychiatry 71 : 525-527, 2001

11. Margalit NS, Lesser JB, Moche J, Sen C : Meningiomas involving the op- tic nerve : technical aspects and outcomes for a series of 50 patients. Neurosurgery 53 : 523-532; discussion 532-533, 2003

12. Mathiesen T, Kihlström L : Visual outcome of tuberculum sellae meningiomas after extradural optic nerve decompression. Neurosurgery 59 : 570-576; discussion 570-576, 2006

13. Narayan S, Cornblath WT, Sandler HM, Elner V, Hayman JA : Preliminary visual outcomes after three-dimensional conformal radiation therapy for optic nerve sheath meningioma. Int J Radiat Oncol Biol Phys $56: 537-543,2003$

14. Panciani PP, Crobeddu E, Garbossa D, Ducati A, Fontanella M : Optic nerve fenestration in sphenoid wing meningioma. Neurol India 59 : 461463, 2011

15. Schick U, Hassler W : Surgical management of tuberculum sellae meningiomas : involvement of the optic canal and visual outcome. J Neurol Neurosurg Psychiatry 76 : 977-983, 2005

16. Takagi Y, Miyamoto S : Penetration of the optic nerve and falciform ligament by an internal carotid artery-ophthalmic artery aneurysm : case report. Neurol Med Chir (Tokyo) $54: 211-213,2014$

17. Wang YY, Thani NB, Han TF : Optic nerve penetration by a caroticoophthalmic artery aneurysm. J Clin Neurosci 17 : 931-933, 2010 